\title{
Proteínas importantes para la invasión de Babesia bovis a las células huésped
}

\author{
Laura Esperanza Cuy-Chaparro ${ }^{(D)}$, Anny Camargo Mancipe ${ }^{2}$ (D) Álida Marcela Gómez Rodríguez², \\ César Reyes Santofimio ${ }^{1}$ (D), Darwin Andrés Moreno Pérez ${ }^{1}$ iD
}

\section{RESUMEN}

Introducción: La babesiosis bovina es causada por parásitos Apicomplexa del género Babesia, siendo la Babesia bovis la especie asociada con cuadros clínicos más graves de la enfermedad. La invasión de B. bovis a los eritrocitos bovinos implica la interacción entre moléculas de los merozoítos del parásito con receptores de las células huésped. Por ende, conocer las proteínas involucradas en este proceso supone un importante paso para entender la biología del parásito.

Objetivo: Describir las principales moléculas implicadas en el proceso de invasión de B. bovis a eritrocitos bovinos. Metodología: Se realizó una búsqueda en NCBI, Medline, LILACS y SciELO usando los términos: "Babesia bovis AND invasion process", "MSA-1", "RON2", "AMA-1", "moving junction", "B. bovis AND Vaccine candidates". Con corte en mayo de 2020, había 61 publicaciones disponibles en inglés que describen el estudio de las anteriores proteínas y su participación en la invasión.

Resultados: Por ser clave el proceso de invasión a eritrocitos bovinos para la patogénesis de la babesiosis bovina, la revisión encontró 3 proteínas de B. bovis que participan en el reconocimiento e invasión a las células diana: MSA-1, AMA-1 y RON2. Sin embargo, los detalles a nivel molecular para las interacciones inter e intramoleculares aún no se han dilucidado por completo.

Conclusiones: Conocer las moléculas involucradas en las interacciones parásito-hospedero permitirá entender cómo ocurre el proceso de invasión de B. bovis a los eritrocitos y, así, evaluar su futura utilidad como componente de una estrategia de control efectiva contra esta parasitosis.

Palabras clave: Babesia bovis; babesiosis; interacciones huésped-parásito; proteínas; control de infección.

${ }^{1}$ Universidad del Rosario, Bogotá, Colombia.

2 Universidad de Boyacá, Tunja, Colombia.

Autora de correspondencia: Laura Cuy-Chaparro. Correo electrónico: lauracuy@outlook.com

Citar este artículo así:

Cuy-Chaparro L, Camargo Mancipe A, Gómez Rodríguez ÁM, Reyes Santofimio C, Moreno Pérez DA. Proteínas importantes para la invasión de Babesia bovis a las células huésped. Rev Investig Salud Univ Boyacá. 2021;8(1):75-90. https://doi.org/10.24267/23897325.475 


\title{
Important Proteins for Babesia bovis Invasion to Host Cells
}

\begin{abstract}
Introduction: Bovine babesiosis is caused by Apicomplexas parasites of the genus Babesia, Babesia bovis being the species associated with the most serious clinical conditions of the disease. $B$. bovis invasion into the bovine erythrocytes involves the interaction between the parasites merozoites molecules with host cell receptors. Therefore, knowing the proteins involved in the invasion process will enable understanding the parasite biology.
\end{abstract}

Objective: To describe the important molecules involved in the B. bovis invasion process to bovine erythrocytes.

Methodology: A search was made on NCBI, Medline, LILACS and SciELO databases using keywords as "Babesia bovis AND invasion process", "MSA-1", "RON2", "AMA-1", "moving junction", "B. bovis AND Vaccine candidates". 61 studies written in English describing the study for proteins that take place during invasion process which have been published until mayo were completely revised.

Results: Given that the bovine erythrocyte invasion process is key for the pathogenesis of bovine babesiosis, a review was made where 3 proteins were found to be associated to the recognition and invasion processes of target cells: MSA-1, AMA-1 and RON2. However, the details at molecular level for the inter an intramolecular interaction have not yet been fully elucidated.

Conclusions: Study the molecules involved in host-parasite interactions will allow understanding how the $B$. bovis invasion process to erythrocytes occurs and evaluating their future utility as a component of an effective control strategy for this parasitosis.

Keywords: Babesia bovis; babesiosis; host-parasite interactions; proteins; infection control. 


\title{
Proteínas importantes para a invasão das células hospedeiras por Babesia bovis
}

\begin{abstract}
RESUMO
Introdução: A babesiose bovina é causada por parasitas Apicomplexa do gênero Babesia, sendo a Babesia bovis a espécie associada com os sinais clínicos mais graves da doença. A invasão de $B$. bovis em eritrócitos bovinos envolve a interação entre moléculas dos merozoítos parasitas com receptores nas células hospedeiras. Por conseguinte, o conhecimento das proteínas envolvidas neste processo é um passo importante para a compreensão da biologia do parasita.
\end{abstract}

Objetivo: Descrever as principais moléculas envolvidas no processo de invasão de $B$. bovis em eritrócitos bovinos.

Metodologia: Foi realizada uma pesquisa no NCBI, Medline, LILACS e SciELO utilizando os termos: "Babesia bovis AND invasion process", "MSA-1", "RON2", "AMA-1", "moving junction", "B. bovis AND Vaccine candidates". Até maio de 2020 estavam disponíveis 61 publicações em inglês, que descreviam o estudo das proteínas acima referidas e o seu envolvimento na invasão.

Resultados: Como o processo de invasão de eritrócitos bovinos é fundamental para á patogênese da babesiose bovina, a revisão encontrou 3 proteínas de $B$. bovis envolvidas no reconhecimento e invasão de células alvo: MSA-1, AMA-1 e RON2. No entanto, os detalhes a nível molecular para as interações Inter e intramoleculares ainda não foram completamente elucidados.

Conclusões: A compreensão das moléculas envolvidas nas interações parasita-hospedeiro permitirá entender como ocorre o processo da invasão de $B$. bovis em eritrócitos e, assim, avaliar sua utilidade futura como componente de uma estratégia efetiva de controle contra esta parasitose.

Palavras-chave: Babesia bovis; babesiose; interações parasita-hospedeiro; proteínas; controle de infecção. 


\section{INTRODUCCIÓN}

La babesiosis ocasionada por hemoprotozoos del género Babesia es una enfermedad veterinaria transmitida por artrópodos que afectan principalmente al ganado bovino, con una proporción de infectados que supera los 1,3 billones de animales (1-3). La Babesia bovis y la Babesia bigemina, los principales agentes causales de la babesiosis (4)are all transmitted by Rhiphicephallus (Boophilus, presentan una amplia distribución en zonas tropicales y subtempladas del mundo y causan distintos cuadros clínicos, aun cuando los de mayor severidad son los de $B$. bovis $(1,5,6)$.

La $B$. bovis tiene un complejo ciclo de vida que involucra el desarrollo de una etapa asexual y una etapa sexual (7) especially the events leading to host switching and diversification, as well as alterations of the life cycle (life-history traits. La primera inicia con la inoculación de esporozoítos por medio de la picadura de la garrapata, los cuales invaden los eritrocitos bovinos. Una vez en su interior, los parásitos se reproducen por fisión binaria y dan lugar a la formación de merozoítos con capacidad infectiva, con lo cual se perpetúa el crecimiento del parásito dentro del hospedero $(8,9)$.

El primer paso del proceso de invasión de merozoítos de Babesia a eritrocitos bovinos consiste en el reconocimiento inicial que se da gracias a la interacción entre proteínas de superficie de los merozoítos babesiales con las moléculas de superficie presentes en las células del hospedero $(10,11)$. Posteriormente, el parásito se reorienta hacia su polo apical para disponer el contenido de las roptrias y micronemas y así establecer una unión móvil (UM) que facilita la invaginación de la membrana y la interiorización del parásito dentro de la célula $(12,13)$. Por lo tanto, conocer las proteínas involucradas en la etapa del contacto inicial y contacto fuerte supone un importante paso para entender la maquinaria de adhesión e invasión del parásito.

En ese orden de ideas, este artículo de revisión presenta aspectos relevantes de las principales moléculas implicadas en el proceso de invasión de la $B$. bovis a eritrocitos bovinos, con el fin de discutir una posible medida de control basada en la información encontrada. Estos datos serán de particular importancia para futuras investigaciones orientadas a profundizar en el papel funcional de las proteínas descritas.

\section{METODOLOGÍA}

Se revisó la literatura sobre el tema para analizar la información existente hasta mayo de 2020, relacionada con la descripción de las moléculas implicadas en el proceso de invasión de la $B$. bovis a eritrocitos bovinos. Se utilizaron fuentes de información primaria como ScienceDirect y Elsevier. 
Las bases de datos consultadas fueron: PubMed del Centro Nacional de Información Biotecnológica (NCBI), Medline, LILACS y SciELO, utilizando diferentes combinaciones de palabras clave en inglés con el operador booleano AND, entre ellas: "(Babesia bovis) AND Erythrocyte invasion", "babesiosis", "MSA-1", "RON2", "AMA-1", "moving junction", "B. bovis AND Vaccine candidates". Se obtuvieron 312 publicaciones en total, de los cuales se seleccionaron 61 artículos científicos disponibles en texto completo en idioma inglés, que además de describir las principales proteínas de $B$. bovis involucradas en la adhesión de los eritrocitos bovinos, también daban evidencia de su participación en el proceso de invasión. No se utilizaron otros criterios de selección, debido a la limitada información existente acerca del parásito.

\section{RESULTADOS}

\section{Dinámica del proceso de invasión en Apicomplexa}

Los parásitos Apicomplexa tienen pasos de invasión muy conservados; dos de los más importantes involucran el contacto inicial y la unión móvil a la célula diana (14). Estos parásitos también se caracterizan por poseer organelos apicales denominados roptrias y micronemas y, además, presentan orgánulos densos que secretan proteínas a la superficie de la membrana de merozoítos o al medio externo para participar en la invasión a sus células diana $(12,15,16)$. Las roptrias son organelos individuales que se dividen en dos compartimentos: un cuerpo bulboso y un cuello estrecho que se extiende por la porción apical del parásito a través del cual se secretan proteínas (17). Por su parte, los micronemas son pequeños organelos tubulares que contienen adhesinas que, al igual que las de roptrias, son secretadas al entrar en contacto con la célula diana para facilitar la motilidad, la unión y la invasión $(18,19)$.

Las principales proteínas implicadas en el contacto inicial con la célula huésped son aquellas localizadas en la superficie del parásito, denominadas antígenos de superficie de merozoíto (Merozoite Surface Antigens [MSA]), las cuales son abundantemente expresadas $y$, por ende, se cree que participan en el contacto inicial y reorientación del parásito hacia su polo apical $(17,20-22)$. Después, participa el antígeno de membrana apical 1 (Apical Marozoite Antigen 1 [AMA-1]), derivado de las micronemas y las proteínas de cuello de roptrias (Roptry Neck Protein [RON]), secretadas por las roptrias (23-25). Estas moléculas forman un complejo proteico que establece la interacción con la membrana celular del hospedero a través de una estructura denominada UM, esencial para el proceso de interiorización del parásito (figura 1). Por último, la membrana de la célula huésped sufre un resellado que deja encerrado al parásito en su interior (26-28). Aunque el proceso de invasión ha sido ampliamente estudiado en Plasmodium y 
Figura 1. Esquema de los pasos iniciales del proceso de invasión de merozoítos (Mrz) de parásitos Apicomplexas a sus células hospederas. Se muestra el contacto inicial que ocurre a través de interacciones intermoleculares entre la proteína MSA1, localizada en la superficie del parásito, y los receptores de las células, seguida de la formación de la UM, que se da por las interacciones intramoleculares entre las proteínas del parásito AMA-1 y RON2.

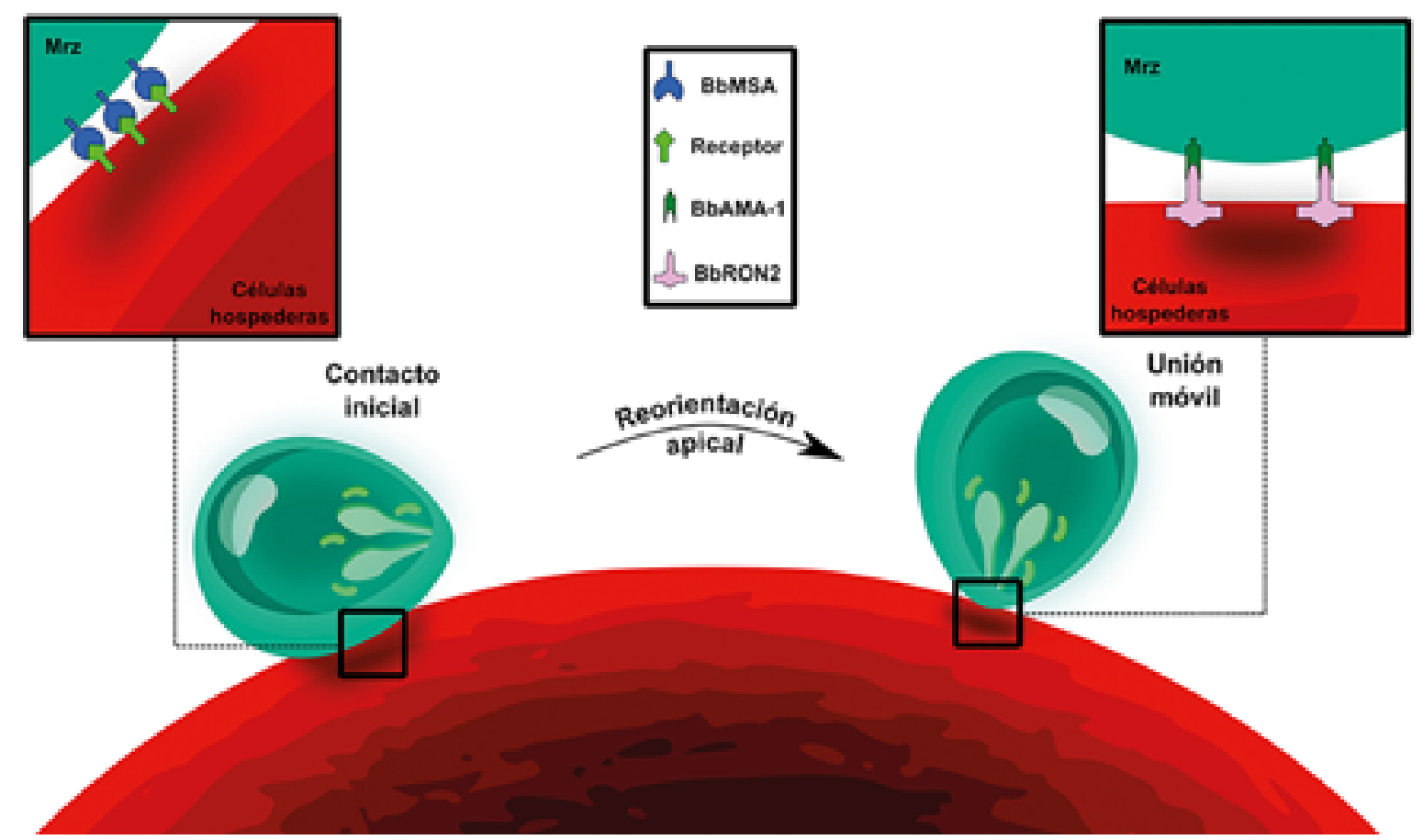

Toxoplasma, en la actualidad, la falta de caracterización de antígenos de $B$. bovis y sus interacciones intermoleculares han limitado el total entendimiento de esta etapa. Sin embargo, se describirán aquellas proteínas homólogas a las mencionadas, que han sido estudiadas en la especie $B$. bovis.

\section{Antígeno de superficie de merozoíto 1 (MSA-1)}

MSA-1 es una glucoproteína de 42 kDa y 319 aminoácidos (aa), codificada por un solo gen que pertenece a la familia de antígenos de superficie de merozoíto variables (variable merozoite surface antigen [VMSA]) $(29,30)$. Esta proteína es abundantemente expresada en parásitos maduros y 
se encuentra distribuida sobre la superficie de merozoítos de $B$. Bovis (31). Además, presenta un anclaje de glucosilfosfatidilinositol (GPI) ubicado en el extremo carboxilo terminal igual que las MSP de otros Apicomplexas, por lo cual se cree que dicha molécula permite la unión inicial del parásito a los eritrocitos bovinos (32-34).

La diversidad genética de MSA-1 presente en cepas de diferentes regiones del mundo ha sido asociada con propiedades de antigenicidad y con una estrategia de supervivencia adoptada por $B$. Bovis $(35,36)$. El polimorfismo de MSA-1 podría estar explicado por: 1) la presión inmune ejercida por el hospedero, 2) un proceso biológico independiente o 3) ambos $(37,38)$. La exposición de una proteína con diversidad antigénica al sistema inmunológico, como es el caso de MSA-1, podría considerarse un mecanismo utilizado por el parásito para evadir la respuesta inmunológica protectora desarrollada en el ganado durante exposiciones previas a $B$. bovis $(34,39)$. Por lo tanto, la variación antigénica que caracteriza a MSA-1 es esencial para llevar a cabo el proceso de invasión (40).

Diferentes estudios han sugerido el papel crítico de MSA-1 en los primeros pasos de la invasión de $B$. bovis a eritrocitos bovinos $(32,33)$. La inmunización de ganado con MSA-1 recombinante induce anticuerpos que neutralizan la invasión de los merozoítos in vitro, que evidencian tan solo un $0,34 \%$ de eritrocitos parasitados a las $96 \mathrm{~h}$.
Sin embargo, estos anticuerpos no confieren protección al ganado que fue sometido a un reto con la cepa virulenta T2BO de B. bovis (32).

La generación de sueros bovinos con anticuerpos dirigidos contra MSA-1 nativo logró neutralizar la infectividad de merozoítos in vitro en un $98 \%$ a las 96 h (33). Adicionalmente, el uso de anticuerpos específicos contra MSA-1 recombinante evidenció la disminución de la unión de esporozoítos a eritrocitos en un $63 \%$ a las 48 h (41). La expresión de MSA-1 tanto en merozoítos como en esporozoítos la ha postulado como un interesante candidata a vacuna, ya que al generar un bloqueo temprano en la ruta de invasión, podría mejorar la efectividad de una vacuna contra $B$. bovis (41). Sin embargo, su uso debería estar asociado a inmunógenos que induzcan una respuesta inmunológica protectiva para prevenir la aparición de babesiosis (32).

\section{Antígeno de membrana apical 1 (AMA-1)}

El AMA-1 de $B$. bovis (BbAM-A1), una proteína transmembrana tipo I codificada por 605 aa y un peso molecular predicho de $66,7 \mathrm{kDa}$, es secretada por micronemas a la superficie del merozoíto y esporozoíto para participar en el proceso de invasión $(28,42,43)$. Esta proteína es conservada dentro del filo Apicomplexa $(42,44)$, ya que comparte tres características estructurales: 1) una región amino-terminal compuesta por un 
péptido señal en los primeros 39 aa y un ectodominio que presenta 8 enlaces disulfuro formados por la asociación entre 16 cisteínas conservadas que pliegan la proteína en los dominios $\mathrm{I}_{(41-271 \text { aa) }}$ $\mathrm{II}_{(272-409 \text { aа) }}$ y III $\mathrm{(410-522} \mathrm{aа);}$ 2) un dominio transmembranal, y 3) una cola citoplasmática en el C-terminal (45-47).

Posterior al contacto inicial y reorientación de los parásitos Apicomplexas hacia su polo apical, AMA-1 es secretada para formar un complejo con las proteínas RON y establecer la UM (24). Aunque en Plasmodium y Toxoplasma ha sido descrita la unión de RON2 al surco hidrofóbico de AMA-1 ubicado en el dominio II $(48,49)$ the Rhoptry Neck Protein (RON, hasta la fecha no se conoce con exactitud la dinámica de interacción entre estas dos moléculas en $B$. bovis.

El estudio de diversidad genética de BbAMA-1 reveló bajos niveles de polimorfismo a lo largo de su secuencia completa, aun cuando los dominios I y III son más polimórficos que el dominio II. Este resultado coincide con análisis de polimorfismo de AMA-1 de Plasmodium falciparum, que ha permitido inferir que estas regiones podrían estar participando en un proceso que garantiza la supervivencia de ambos parásitos $(50,51)$.

Los diferentes trabajos realizados en Toxoplasma, Plasmodium y Babesia han demostrado cómo la presencia de mutaciones en AMA-1 y el uso de anticuerpos contra esta proteína pueden disminuir la capacidad de invasión del parásito a sus células diana (52-54). En B. bovis, la generación de anticuerpos contra 3 péptidos sintéticos derivados de la región N-terminal y el dominio II de AMA-1 redujo la invasión a los eritrocitos en un $65 \%$ (55). Por otra parte, se ha demostrado el importante papel de la región central de BbAMA-1 (que involucra los dominios I y II), al evidenciar cómo anticuerpos dirigidos contra esta región logran inhibir la invasión a eritrocitos en un $61 \%$ y un $70 \%$ a las $3 \mathrm{~h}$ y $6 \mathrm{~h}$, respectivamente (46). En conjunto, estos resultados destacan que posiblemente BbAMA-1 desempeña un rol primordial en el proceso de invasión de $B$. bovis a eritrocitos bovinos $y$, por ende, destaca su importancia para postularlo como un antígeno candidato a vacuna.

\section{Proteína de cuello de roptrias 2 (RON2)}

RON2 es una proteína de 1365 aa con 150 kDa de masa molecular, codificada por un solo gen y localizada en la región del cuello de las roptrias. Esta molécula posee un péptido señal ubicado en los primeros 19 aa, tres dominios transmembrana y un dominio tipo cytoadherence linked asexual gene (CLAG) entre los aa 176 y 1168 , que se encuentran implicados en el proceso de citoadherencia de Apicomplexas $(10,56,57)$.

El establecimiento de la UM durante el proceso de invasión de Apicomplexas requiere la asociación 
de AMA-1 y RON2, donde RON-2, a través de su dominio CLAG, se inserta en la membrana de la célula huésped para asegurar una unión estrecha e irreversible $(24,58)$. Adicionalmente, en Plasmodium y Toxoplasma se incluyen en este complejo las proteínas RON4, RON5 y RON8 (59-61), las cuales no han sido del todo caracterizadas en Babesia.

Análisis de identidad realizados con secuencias de aminoácidos de RON2, derivadas de cuatro cepas distintas de $B$. bovis y otras especies como $B$. bigemina, $B$. divergens, B. microti, Theileria equi y $P$. falciparum, revelaron un alto grado de conservación, entre un $99,78 \%$ y un $26 \%$, lo que sugiere su participación en procesos esenciales para la supervivencia de $B$. bovis, por ejemplo, la invasión de eritrocitos bovinos (10). Esta hipótesis se confirmó a través de ensayos de neutralización in vitro usando anticuerpos anti-BbRON2 generados contra péptidos epítopes de células $B$. El estudio permitió confirmar que los anticuerpos eran capaces de bloquear la invasión de merozoítos de $B$. bovis hasta en un $43 \%$ durante $72 \mathrm{~h}$ (10). Considerando estos datos, se ha propuesto a RON2 como una molécula con potencial para ser estudiada como candidata a vacuna contra $B$. bovis.

\section{CONCLUSIÓN}

La invasión de los parásitos Apicomplexa a sus células diana es un proceso bastante conservado que comprende las etapas de contacto inicial, la reorientación y la invasión, facilitada por proteínas que establecen las interacciones específicas receptor-ligando. Toxoplasma gondii y Plasmodium falciparum han sido las especies con mayor número de estudios relacionados con proteínas que participan en el proceso de invasión celular. Estas especies tienen proteínas de superficie muy variables que se unen a las 3 principales glucoforinas (GPA, GPB y GPC) y al receptor Banda 3; mientras que presentan otras proteínas como AMA-1 y RON, cuya funcionalidad es muy conservada, dado que ayudan a establecer la unión fuerte entre el parásito y su célula diana. En el caso de Babesia, este mecanismo es poco conocido, dada la poca investigación en este campo. Aunque en la última década diferentes estudios in vitro e in vivo han revelado características de la dinámica de invasión como el tiempo de duración, los efectos sobre la membrana del eritrocito y el papel de algunos ligandos liberados por organelos apicales en especies como $B$. divergens y $B$. microti, no se conoce por completo cómo pueden llegar a participar en el proceso de invasión. Sin embargo, los pocos estudios de caracterización descritos a la fecha para las proteínas de $B$. bovis han permitido sugerir el posible rol en el contacto inicial para MSA-1 y en la unión móvil para AMA-1 y RON2, aunque son desconocidos los receptores para estas moléculas. Así, teniendo claro el rol que representan las anteriores moléculas para el reconocimiento, la motilidad, la reorientación y la interiorización de parásitos Apicomplexa dentro de sus células 
diana, es importante profundizar su estudio en la especie Babesia bovis. Por lo tanto, estudiar moléculas involucradas en estos pasos contribuiría al entendimiento de la biología de $B$. bovis y al desarrollo de estrategias de control efectivas contra esta parasitosis.

\section{CONFLICTO DE INTERESES}

Los autores declaran que no tienen conflictos de intereses.

\section{FINANCIACIÓN}

Este trabajo fue financiado por la Universidad de Boyacá (Colombia).

\section{CONTRIBUCIÓN DE LOS AUTORES}

LC, AC y MG escribieron el borrador inicial. DAMP realizó la revisión crítica del manuscrito. CR modeló la figura. Todos los autores han leído y aprobado la versión final del manuscrito.

\section{AGRADECIMIENTOS}

Queremos agradecer a la Fundación Instituto de Inmunología de Colombia, por permitirnos desarrollar este trabajo en la Línea de Investigación Básica en Biología Molecular.

\section{REFERENCIAS}

1. Bock $R$, Jackson $L$, De Vos $A$, Jorgensen W. Babesiosis of cattle. Parasitology. 2004;129(7):S247-69. https://doi. org/10.1017/S0031182004005190

2. Lew-Tabor $A E$, Rodriguez Valle $M$. A review of reverse vaccinology approaches for the development of vaccines against ticks and tick borne diseases. Ticks Tick-Borne Dis. 2016;7(4):573-85. https://doi.org/10.1016/j. ttbdis.2015.12.012

3. Hunfeld K, Hildebrandt A, Gray J. Babesiosis: recent insights into an ancient disease. Int J Parasitol. 2008;38(11):1219-37. https://doi.org/10.1016/j.ijpara.2008.03.001

4. Suarez $C E$, Noh $S$. Emerging perspectives in the research of bovine babesiosis and anaplasmosis. Vet Parasitol. 2011;180(1-2):109-25. https://doi.org/10.1016/j.vetpar.2011.05.032

5. Ishizaki T, Sivakumar T, Hayashida K, Tuvshintulga B, Igarashi I, Yokoyama N. RBC invasion and invasion-inhibition assays using free merozoites isolated after cold treatment of Babesia bovis in vitro culture. Exp Parasitol. 2016;166:10-5. https://doi.org/10.1016/j. exppara.2016.03.010 
6. Nava A, Venzal J, González-Acuña D, Martins T, Guglielmone A. Ticks of the Southern Cone of America: diagnosis, distribution, and hosts with taxonomy, ecology and sanitary importance. New York: Academic Press; 2017.

7. Martinsen ES, Perkins SL, Schall JJ. A three-genome phylogeny of malaria parasites (Plasmodium and closely related genera): evolution of life-history traits and host switches. Mol Phylogenet Evol. 2008;47(1):261-73. https:// doi.org/10.1016/j.ympev.2007.11.012

8. Lobo CA, Rodriguez $M$, Cursino-Santos JR. Babesia and red cell invasion: Curr Opin Hematol. 2012;19(3):170-5. https://doi. org/10.1097/moh.0b013e328352245a

9. Chauvin A, Moreau E, Bonnet S, Plantard O, Malandrin L. Babesia and its hosts: adaptation to long-lasting interactions as a way to achieve efficient transmission. Vet Res. 2009;40(2):37. https://doi.org/10.1051/vetres/2009020

10. Hidalgo-Ruiz $M$, Suárez $C E$, Mercado-Uriostegui MA, Hernandez-Ortiz R, Ramos JA, Galindo-Velasco $E$, et al. Babesia bovis RON2 contains conserved B-cell epitopes that induce an invasion-blocking humoral immune response in immunized cattle. Parasit Vectors. 2018;11(1):575. https://doi.org/10.1186/ s13071-018-3164-2
11. Kwong WK, del Campo J, Mathur V, Vermeij MJA, Keeling PJ. A widespread coral-infecting apicomplexan contains a plastid encoding chlorophyll biosynthesis. bioRxiv. 2018. https://doi.org/10.1101/391565

12. Dubremetz JF, Garcia-Réguet N, Conseil V, Fourmaux MN. Apical organelles and host-ceII invasion by Apicomplexa. Int J Parasitol. 1998;28(7):1007-13. https://doi.org/10.1016/ S0020-7519(98)00076-9

13. Yokoyama N, Okamura M, Igarashi I. Erythrocyte invasion by Babesia parasites: current advances in the elucidation of the molecular interactions between the protozoan ligands and host receptors in the invasion stage. Vet Parasitol. 2006;138(1-2):22-32. https://doi.org/10.1016/j.vetpar.2006.01.037

14. Bargieri D, Lagal V, Andenmatten N, Tardieux I, Meissner M, Ménard R. Host cell invasion by apicomplexan parasites: the junction conundrum. PLoS Pathog. 2014 sep;10(9):e1004273. https://doi.org/10.1371/journal.ppat.1004273

15. Proellocks NI, Coppel RL, Waller KL. Dissecting the apicomplexan rhoptry neck proteins. Trends Parasitol. 2010;26(6):297-304. https:// doi.org/10.1016/j.pt.2010.02.012

16. Tyler JS, Treeck M, Boothroyd JC. Focus on the ringleader: the role of AMA1 in apicomple- 
xan invasion and replication. Trends Parasitol. 2011;27(9):410-20. https://doi.org/10.1016/j. pt.2011.04.002

17. Bradley PJ, Ward C, Cheng SJ, Alexander $\mathrm{DL}$, Coller S, Coombs GH, et al. Proteomic analysis of rhoptry organelles reveals many novel constituents for host-parasite interactions in Toxoplasma gondii. J Biol Chem. 2005;280(40):34245-58. https://doi. org/10.1074/jbc.M504158200

18. Morrissette NS, Sibley LD. Cytoskeleton of apicomplexan parasites. Microbiol Mol Biol Rev MMBR. 2002;66(1):21-38. https://doi. org/10.1128/MMBR.66.1.21-38.2002

19. Portman N, Foster C, Walker G, Šlapeta J. Evidence of intraflagellar transport and apical complex formation in a free-living relative of the apicomplexa. Eukaryot Cell. 2014;13(1):1020. https://doi.org/10.1128/EC.00155-13

20. Carruthers VB, Sibley LD. Sequential protein secretion from three distinct organelles of Toxoplasma gondii accompanies invasion of human fibroblasts. Eur J Cell Biol. 1997;73(2):114-23.

21. Bradley PJ, Sibley LD. Rhoptries: an arsenal of secreted virulence factors. Curr Opin Microbiol. 2007;10(6):582-7. https://doi.org/10.1016/j.mib.2007.09.013
22. Woehlbier U, Epp C, Hackett F, Blackman MJ, Bujard $\mathrm{H}$. Antibodies against multiple merozoite surface antigens of the human malaria parasite Plasmodium falciparum inhibit parasite maturation and red blood cell invasion. Malar J. 2010;9:77. https://doi.org/10.1186/14752875-9-77

23. Srinivasan $P$, Beatty $W L$, Diouf $A$, Herrera $\mathrm{R}$, Ambroggio $X$, Moch JK, et al. Binding of Plasmodium merozoite proteins RON2 and AMA1 triggers commitment to invasion. Proc Natl Acad Sci U S A. 2011;108(32):13275-80. https://doi.org/10.1073/pnas.1110303108

24. Lamarque $M$, Besteiro S, Papoin J, Roques M, Vulliez-Le Normand B, Morlon-Guyot J, et al. The RON2-AMA1 interaction is a critical step in moving junction-dependent invasion by apicomplexan parasites. PLoS Pathog. 2011;7(2):e1001276. https://doi.org/10.1371/ journal.ppat.1001276

25. Lebrun $M$, Michelin A, El Hajj $H$, Poncet $J$, Bradley PJ, Vial $\mathrm{H}$, et al. The rhoptry neck protein RON4 relocalizes at the moving junction during Toxoplasma gondii invasion. Cell Microbiol. 2005;7(12):1823-33. https://doi.org/10.1111/j.1462-5822.2005.00646.x

26. Shen B, Sibley LD. The moving junction, a key portal to host cell invasion by api- 
complexan parasites. Curr Opin Microbiol. 2012;15(4):449-55. https://doi.org/10.1016/j. mib.2012.02.007

27. Besteiro S, Michelin A, Poncet J, Dubremetz J-F, Lebrun M. Export of a Toxoplasma gondii rhoptry neck protein complex at the host cell membrane to form the moving junction during invasion. PLoS Pathog. 2009;5(2):e1000309. https://doi.org/10.1371/journal.ppat.1000309

28. Besteiro S, Dubremetz J-F, Lebrun M. The moving junction of apicomplexan parasites: a key structure for invasion: The moving junction of apicomplexan parasites. Cell Microbiol. 2011;13(6):797-805. https://doi.org/10.1111/ j.1462-5822.2011.01597.x

29. Hines S, Mcelwain T, Buening G, Palmer G. Molecular characterization of Babesia bovis merozoite surface proteins bearing epitopes immunodominant in protected cattle. Mol Biochem Parasitol. 1989;37(1):1-9. https://doi. org/10.1016/0166-6851(89)90096-0

30. Goff WL, Davis WC, Palmer GH, McElwain $\mathrm{TF}$, Johnson WC, Bailey JF, et al. Identification of Babesia bovis merozoite surface antigens by using immune bovine sera and monoclonal antibodies. Infect Immun. 1988;56(9):2363-8. https://doi.org/10.1128/iai.56.9.2363-2368.1988
31. Johnson WC, Taus NS, Reif KE, Bohaliga GA, Kappmeyer LS, Ueti MW. Analysis of stage-specific protein expression during Babesia bovis development within female rhipicephalus microplus. J Proteome Res. 2017;16(3):1327-38. https://doi.org/10.1021/acs.jproteome.6b00947

32. Hines SA, Palmer GH, Jasmer DP, Goff WL, McElwain TF. Immunization of cattle with recombinant Babesia bovis merozoite surface antigen-1. Infect Immun. 1995;63(1):349-52. https://doi.org/10.1128/iai.63.1.349-352.1995

33. Hines SA, Palmer GH, Jasmer DP, McGuire TC, McElwain TF. Neutralization-sensitive merozoite surface antigens of Babesia bovis encoded by members of a polymorphic gene family. Mol Biochem Parasitol. 1992;55(1-2):85-94. https:// doi.org/10.1016/0166-6851(92)90129-8

34. Carcy B, Précigout E, Schetters T, Gorenflot A. Genetic basis for GPI-anchor merozoite surface antigen polymorphism of Babesia and resulting antigenic diversity. Vet Parasitol. 2006;138(1-2):33-49. https://doi.or$\mathrm{g} / 10.1016 / \mathrm{j}$.vetpar.2006.01.038

35. Deitsch KW, Lukehart SA, Stringer JR. Common strategies for antigenic variation by bacterial, fungal and protozoan pathogens. Nat Rev Microbiol. 2009;7(7):493-503. https:// doi.org/10.1038/nrmicro2145 
36. Genis AD, Mosqueda JJ, Borgonio VM, Falcón A, Alvarez A, Camacho M, et al. Phylogenetic analysis of Mexican Babesia bovis isolates using msa and ssrRNA gene sequences. Ann N Y Acad Sci. 2008;1149:121-5. https://doi. org/10.1196/annals.1428.070

37. Leroith T, Brayton KA, Molloy JB, Bock RE, Hines $S A$, Lew $A E$, et al. Sequence variation and immunologic cross-reactivity among Babesia bovis merozoite surface antigen 1 proteins from vaccine strains and vaccine breakthrough isolates. Infect Immun. 2005;73(9):5388-94. https:// doi.org/10.1128/IAI.73.9.5388-5394.2005.

38. Tattiyapong $M$, Sivakumar $T$, Takemae $H$, Simking $P$, Jittapalapong $S$, Igarashi $I$, et al. Genetic diversity and antigenicity variation of Babesia bovis merozoite surface antigen-1 (MSA-1) in Thailand. Infect Genet Evol J Mol Epidemiol Evol Genet Infect Dis. 2016;41:255-61. https://doi.org/10.1016/j. meegid.2016.04.021

39. Sivakumar T, Okubo K, Igarashi I, de Silva WK, Kothalawala H, Silva SSP, et al. Genetic diversity of merozoite surface antigens in Babesia bovis detected from Sri Lankan cattle. Infect Genet Evol J Mol Epidemiol Evol Genet Infect Dis. 2013;19:134-40. https://doi.org/10.1016/j. meegid.2013.07.001
40. Suarez $C E$, Florin-Christensen $M$, Hines $S A$, Palmer GH, Brown WC, McElwain TF. Characterization of Allelic Variation in the Babesia bovis Merozoite Surface Antigen 1 (MSA-1) locus and identification of a cross-reactive inhibition-sensitive MSA-1 epitope. Infect Immun. 2000;68(12):6865-70. https://doi. org/10.1128/IAl.68.12.6865-6870.2000

41. Mosqueda J. McElwain TF. Stiller D. Palmer $\mathrm{GH}$. Babesia bovis merozoite surface antigen 1 and rhoptry-associated protein 1 are expressed in sporozoites, and specific antibodies inhibit sporozoite attachment to erythrocytes. Infect Immun. 2002;70(3):1599-603. https:// doi.org/10.1128/IAl.70.3.1599-1603.2002

42. Triglia T, Healer J, Caruana SR, Hodder AN, Anders RF, Crabb BS, et al. Apical membrane antigen 1 plays a central role in erythrocyte invasion by Plasmodium species. Mol Microbiol. 2000;38(4):706-18. https://doi.org/10.1046/ j.1365-2958.2000.02175.x

43. Hodder AN, Crewther PE, Anders RF. Specificity of the protective antibody response to apical membrane antigen 1. Infect Immun. 2001;69(5):3286-94. https://doi.org/10.1128/ IAl.69.5.3286-3294.2001

44. Hehl AB, Lekutis C, Grigg ME, Bradley PJ, Dubremetz J-F, Ortega-Barria E, et al. Toxoplasma 
gondii homologue of plasmodium apical membrane antigen 1 is involved in invasion of host cells. Infect Immun. 2000;68(12):7078-86. https:// doi.org/10.1128/IAl.68.12.7078-7086.2000

45. Montero E, Rodríguez M, Oksov Y, Lobo CA. Babesia divergens apical membrane antigen 1 and its interaction with the human red blood cell. Infect Immun. 2009;77(11):4783-93. https://doi.org/10.1128/IAI.00969-08

46. Salama AA, Terkawi MA, Kawai S, AbouLaila $M$, Nayel $M$, Mousa $A$, et al. Specific antibody to a conserved region of Babesia apical membrane antigen-1 inhibited the invasion of B. bovis into the erythrocyte. Exp Parasitol. 2013;135(3):623-8. https://doi.org/10.1016/j. exppara.2013.09.017

47. Remarque EJ, Faber BW, Kocken CHM, Thomas AW. Apical membrane antigen 1: a malaria vaccine candidate in review. Trends Parasitol. 2008;24(2):74-84. https://doi.org/10.1016/j. pt.2007.12.002

48. Delgadillo RF, Parker ML, Lebrun M, Boulanger MJ, Douguet D. Stability of the Plasmodium falciparum AMA1-RON2 complex is governed by the domain II (DII) loop. PloS One. 2016;11(1):e0144764. https://doi. org/10.1371/journal.pone.0144764
49. Tyler JS, Boothroyd JC. The C-terminus of toxoplasma RON2 provides the crucial link between AMA1 and the host-associated invasion complex. PLoS Pathog. 2011;7(2):e1001282. https://doi.org/10.1371/journal.ppat.1001282

50. Rittipornlertrak A, Nambooppha B, Simking P, Punyapornwithaya V, Tiwananthagorn S, Jittapalapong $S$, et al. Low levels of genetic diversity associated with evidence of negative selection on the Babesia bovis apical membrane antigen 1 from parasite populations in Thailand. Infect Genet Evol. 2017;54:447-54. https://doi.org/10.1016/j.meegid.2017.08.009

51. Polley SD, Conway DJ. Strong diversifying selection on domains of the Plasmodium falciparum apical membrane antigen 1 gene. Genetics. 2001;158(4):1505-12.

52. Mital J, Meissner M, Soldati D, Ward GE. Conditional expression of Toxoplasma gondii apical membrane antigen-1 (TgAMA1) demonstrates that TgAMA1 plays a critical role in host cell invasion. Mol Biol Cell. 2005;16(9):4341-49. https://doi.org/10.1091/mbc.e05-04-0281

53. Yap A, Azevedo MF, Gilson PR, Weiss GE, O'Neill MT, Wilson DW, et al. Conditional expression of apical membrane antigen 1 in Plasmodium falciparum shows it is required for erythrocyte invasion by merozoites. Cell 
Microbiol. 2014;16(5):642-56. https://doi. org/10.1111/cmi.12287

54. Bilgic HB, Hacilarlioglu S, Bakirci S, Kose O, Unlu AH, Aksulu A, et al. Comparison of protectiveness of recombinant Babesia bovis apical membrane antigen 1 and B. ovis-infected cell line as vaccines against ovine babesiosis. Ticks Tick-Borne Dis. 2020;11(1):101280. https://doi.org/10.1016/j.ttbdis.2019.101280

55. Gaffar FR, Yatsuda AP, Franssen FFJ, de Vries E. Erythrocyte invasion by Babesia bovis merozoites is inhibited by polyclonal antisera directed against peptides derived from a homologue of Plasmodium falciparum apical membrane antigen 1. Infect Immun. 2004;72(5):2947-55. https://doi. org/10.1128/IAl.72.5.2947-2955.2004

56. Gardiner DL, Spielmann T, Dixon MWA, Hawthorne $\mathrm{PL}$, Ortega $\mathrm{MR}$, Anderson $\mathrm{KL}$, et al. CLAG 9 is located in the rhoptries of Plasmodium falciparum. Parasitol Res. 2004;93(1):647. https://doi.org/10.1007/s00436-004-1098-4

57. Kaneko O, Tsuboi T, Ling IT, Howell S, Shirano M, Tachibana M, et al. The high molecular mass rhoptry protein, RhopH1, is encoded by members of the clag multigene family in Plasmodium falciparum and Plasmodium yoelii. Mol Biochem Parasitol. 2001;118(2):223-31. https://doi.org/10.1016/ s0166-6851(01)00391-7
58. Cao J, Kaneko O, Thongkukiatkul A, Tachibana $M$, Otsuki $H, G a o$, et al. Rhoptry neck protein RON2 forms a complex with microneme protein AMA1 in Plasmodium falciparum merozoites. Parasitol Int. 2009;58(1):29-35. https://doi.org/10.1016/j.parint.2008.09.005

59. Morahan BJ, Sallmann GB, Huestis R, Dubljevic $\mathrm{V}$, Waller KL. Plasmodium falciparum: genetic and immunogenic characterisation of the rhoptry neck protein PfRON4. Exp Parasitol. 2009;122(4):280-8. https://doi.org/10.1016/j. exppara.2009.04.013

60. Mutungi JK, Yahata K, Sakaguchi $M$, Kaneko O. Expression and localization of rhoptry neck protein 5 in merozoites and sporozoites of Plasmodium yoelii. Parasitol Int. 2014;63(6):794-801. https://doi.org/10.1016/j.parint.2014.07.013

61. Straub KW, Peng ED, Hajagos BE, Tyler JS, Bradley PJ. The moving junction protein RON8 facilitates firm attachment and host cell invasion in Toxoplasma gondii. PLoS Pathog. 2011;7(3):e1002007. https://doi.org/10.1371/ journal.ppat.1002007

Esta obra está bajo una licencia de Creative Commons Reconocimiento-NoComercial 4.0 Internacional 